\title{
Trapping Effect of Eugenol on Hydroxyl Radicals Induced by L-DOPA in Vitro
}

\author{
Masahiro OGata $,{ }^{*}, a, c$ Daisuke Kaneya,${ }^{b}$ Kazuo Shin-ya ${ }^{d}$ Linxang Li,${ }^{b}$ Yoshihiro Abe,${ }^{b}$ \\ Hironobu KatoH, ${ }^{d}$ Sachiko SeKi, ${ }^{a}$ Yasuhide SeKI, ${ }^{a}$ Ryoko Gonda, ${ }^{b}$ Shiro Urano, ${ }^{c}$ and \\ Toyoshige $\mathrm{ENDO}^{b}$ \\ ${ }^{a}$ Aomori University; 2-3-1 Kohbata, Aomori, Aomori 030-0943, Japan: ${ }^{b}$ Kyoritsu University of Pharmacy; 1-5-30 \\ Shibakoen, Minato-ku, Tokyo 105-8512, Japan: ' Shibaura Institute of Technology; 3-9-14 Shibaura, Minato-ku, Tokyo \\ 108-8548, Japan: and ${ }^{d}$ The University of Tokyo; 1-1-1 Yayoi, Bunkyo-ku, Tokyo 113-0032, Japan.
}

Received February 3, 2005; accepted May 25, 2005

\begin{abstract}
Many researchers have stated that eugenol might inhibit lipid peroxidation at the stage of initiation, propagation, or both, and many attempts have been made to elucidate the mechanism of its antioxidant activity. Nevertheless, details of its mechanism are still obscure. This study was carried out to investigate the trapping effect of eugenol on hydroxyl radical generated from L-3,4-dihydroxyphenylalanine (DOPA) in MiliQ water and the generation mechanism of the hydroxyl radical by this system which uses no metallic factor. This was studied by adding L-DOPA and 5,5-dimethyl-1-pyrroline $N$-oxide (DMPO) to phosphate buffered saline (PBS) or MiliQ water, and the generation of hydroxyl radical was detected on an ESR spectrum. By this method, the effect of antioxidants was detected as a modification of ESR spectra. We found that the eugenol trapped hydroxyl radicals directly, because it had no iron chelating action, did not trap L-DOPA semiquinone radical and inhibited hydroxyl radicals with or without iron ion.
\end{abstract}

Key words L-3,4-dihydroxyphenylalanine (DOPA); eugenol; hydroxyl radical; ESR

It is believed that free radicals may attack several organs to induce aging and many disorders, including cancer, diabetes and arteriosclerosis. ${ }^{1-5)}$ This theory, based on the action of the free radicals, is supported by a great deal of research. Free radicals and other oxidants produced at sites of inflammation will, if not controlled, attack the tissues of the host. In addition, metal-containing proteins, for example hemoglobin, are released from lysed erythrocytes at sites of inflammation, and the iron or other metals contained in these proteins can react with free radicals and reactive oxygen species (ROS) through a series of reactions called the modified Haber-Weiss or Fenton reaction, to generate other, more toxic, oxidant species such as hydroxyl radicals. ${ }^{6-9)}$

The hydroxyl radical $(\cdot \mathrm{OH})$ has a high and indiscriminate activity and can slowly cause severe damage to susceptible biomolecules. Hydroxyl radicals can be produced in cells by a variety of processes: phagocytosis; prostaglandin biosynthesis, especially during the transformation of PGG2 to PGH2; by antineoplastic agents, e.g. bleomycin and adriamycin; fungal phytotoxins, cerosporin and dothistoromin; ionizing irradiation; and decomposition of lipid hydroperoxide. ${ }^{10-16)}$ It is generally proposed that such a radical could be originated by the Fenton-type reaction: $\mathrm{H}_{2} \mathrm{O}_{2}+\mathrm{Fe}^{2+} \rightarrow \cdot \mathrm{OH}+$ $\mathrm{OH}^{-}+\mathrm{Fe}^{3+} .{ }^{17)}$

Eugenol (4-allyl-2-methoxyphenol), a naturally occurring phenol in oils of clove, cinnamon, basil, nutmeg and so on, is reported to show antioxidant activity (Fig. 1). ${ }^{18,19)}$ In a previ-

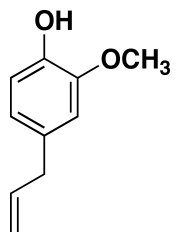

ous paper, we studied the antioxidant activity of eugenol and its related compounds, and found that the mechanism inhibiting lipid peroxidation has two steps: 1) it interferes with the chain reactions by trapping the active oxygen, such as superoxide anion and hydroxyl radicals, 2) it is metabolized to a dimer, and the dimeric compound (dieugenol) inhibits lipid peroxidation at the level of propagation of free radical chain reaction like $\alpha$-tocopherol. ${ }^{20)}$ However, the peroxidation was induced by $\mathrm{Fe}^{2+}$-ascorbic acid system, and the Fenton reaction was used in the generation of the hydroxyl radical. There remains some possibility of suppressing the reaction by chelate formation, with iron in both systems. In this paper, we examined: 1) the chelate formation ability of eugenol and 2) the effect of eugenol on hydroxyl radical in a system which does not contain any metals.

\section{Experimental}

Chemicals Eugenol was obtained from Tokyo Kasei Kogyo Co. (Tokyo, Japan). 5,5-Dimethyl-1-pyrroline $N$-oxide (DMPO) was from Labotec Co. (Tokyo). L-3,4-dihydroxyphenylalanine (DOPA) and $\mathrm{FeSO}_{4}$ were from Wako Pure Chemical Co. (Osaka, Japan).

Iron Chelating Iron chelation was determined by adding $200 \mu \mathrm{m}$ of $\mathrm{FeSO}_{4}$ to the solution containing $200 \mu \mathrm{M}$ of test compounds. After $6.5 \mathrm{~min}$ and $24 \mathrm{~h}$, the change in spectrum by adding the iron was observed by a UV/visible light spectrometer $(190-900 \mathrm{~nm}$, Hitachi 220A (Hitachi Seisakusho Co., Ltd., Japan)). ${ }^{21)}$

Measurement of Hydroxyl Radical ESR spectra were recorded on a JEOL-JES-RE1X spectrometer (JEOL Co., Ltd., Tokyo). Hydroxyl radicals were generated by autoxidation of L-DOPA. Two hundred micromolar LDOPA and DMPO were added to phosphate buffered saline (PBS) or MiliQ water and the reaction mixture was incubated at $37^{\circ} \mathrm{C}$ for $24 \mathrm{~h}$. DMPO-OH spin adducts are obtained when hydroxyl radicals are trapped, so these radical scavenging activities of samples could be calculated as the decreasing rate of the DMPO-OH signal intensities. The ESR conditions were: power, $4 \mathrm{~mW}$; magnetic field, $336.0 \mathrm{mT}$; modulation amplitude, $0.1 \mathrm{mT}$; response time, $0.03 \mathrm{~s}$; receive gain, $\times 500$; sweep time, $0.5 \mathrm{~min}$; and sweep width, $\pm 5 \mathrm{mT}$.

Fig. 1. Structure of Eugenol 
(A)

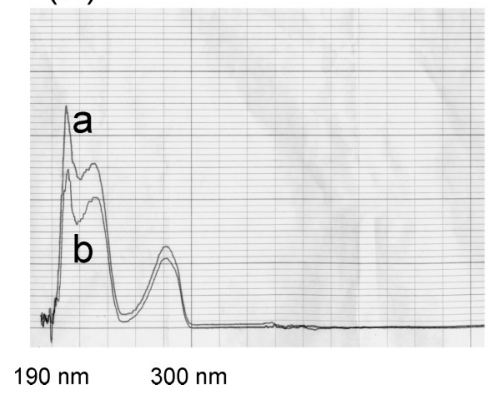

(B)

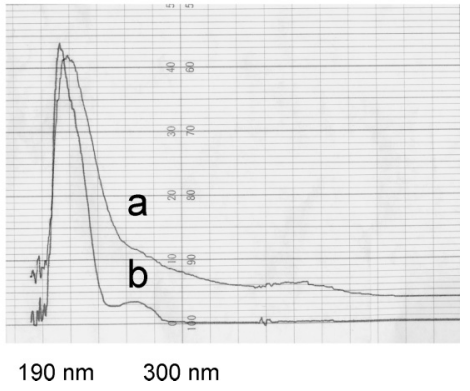

Fig. 2. Chelate Action of Eugenol (A) and Pyrogallol (B)

(a) Only eugenol $(200 \mu \mathrm{M})$ or pyrogallol $(200 \mu \mathrm{M})$; (b) eugenol (or pyrogallol) $(200 \mu \mathrm{M})+\mathrm{FeSO}_{4}(200 \mu \mathrm{M})$, after $24 \mathrm{~h}$.

Table 1. Chelate Action of Eugenol

\begin{tabular}{lc}
\hline \hline Compound & Chelate action \\
\hline Eugenol & - \\
Isoeugenol & - \\
Caffeic acid & + \\
Pyrogallol & + \\
\hline
\end{tabular}

$(+)$ yes, $(-)$ no.

\section{Results and Discussion}

As shown in Table 1 and Fig. 2, iron chelation was determined by adding $200 \mu \mathrm{M}$ of $\mathrm{Fe}^{2+}$ to the solution containing $200 \mu \mathrm{M}$ of test compounds. After $6.5 \mathrm{~min}$ and $24 \mathrm{~h}$, the change of the spectrum by adding the iron was observed with a UV/visible light spectrometer $(190-900 \mathrm{~nm})$. The addition of $\mathrm{Fe}^{2+}$ did not cause a spectral shift or absorbance change in the eugenol or isoeugenol, and chelate formation of these compounds could not be estimated. ${ }^{21)}$

We studied the generation system of the hydroxyl radical which contains no metal. L-DOPA, which has been thought to be an effective drug in Parkinson's disease (PD) therapy, is now believed to be recognized as a worsening factor of this condition because of its generation of ROS. Free radicals are generated during all stages of DOPA polymerization to melanins; for this reason, we examined the generation of ROS using L-DOPA. ${ }^{22)}$ Incubation containing L-DOPA and DMPO, a spin trap reagent, in the PBS or MiliQ water under aerobic conditions at $37^{\circ} \mathrm{C}$ yielded a four-line ESR spectrum characteristic of the DMPO-OH spin adducts $\left(a^{\mathrm{N}}=14.96 \mathrm{G}\right.$, $a^{\mathrm{H}}=14.72 \mathrm{G}$ ) in a time-dependent manner (Figs. 3B-D). There remains a possibility that the metal is included for reagents, etc., and the hydroxyl radical may be generated as the Fenton type reaction. Then, though deferoxamine, which is a chelating reagent, was added to an aqueous solution containing L-DOPA and DMPO, the signal did not disappear (Fig. 3E). ${ }^{23)}$ If DMPO-OH is produced by the free radical mechanism, its ESR signal must be decreased by adding scavengers for hydroxyl radicals. In the present study, therefore, ethanol, which is a hydroxyl radical scavenger, was added to aqueous solution containing L-DOPA and DMPO. As shown in Figs. $3 \mathrm{~F}$ and $\mathrm{G}$, a set of lines due to DMPO- $\mathrm{CH}_{3} \mathrm{CHOH}\left(a^{\mathrm{N}}=16.01 \mathrm{G}, a^{\mathrm{H}}=23.07 \mathrm{G}\right)$ appeared in the ESR spectra and the ESR signal of DMPO- $\mathrm{CH}_{3} \mathrm{CHOH}$ increased and that of DMPO-OH decreased with increase in the concentration of ethanol. ${ }^{24)}$

As DMPO-OH could be generated from the reaction of
(A)

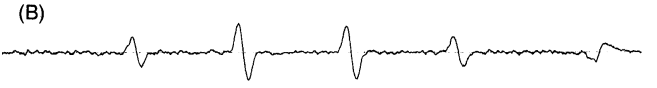

(C)
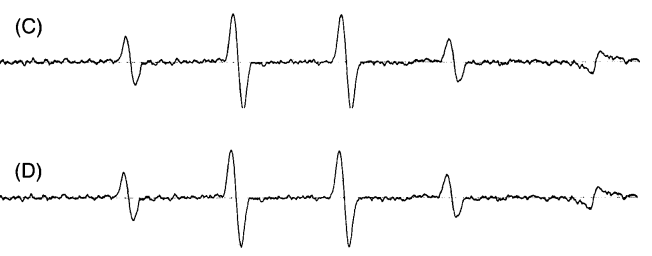

(E)

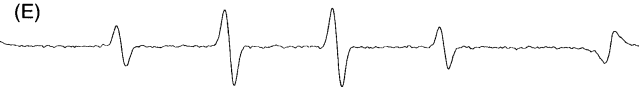

(F)

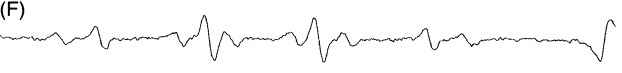

(G)

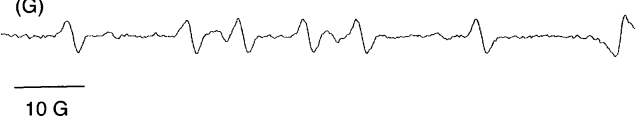

Fig. 3. ESR Spectra of Hydroxyl Radicals Produced by Incubation of LDOPA with Spin-Trapped 5,5-dimethyl-1-pyrroline- $N$-oxide (DMPO)

(A) L-DOPA alone (in PBS); (B) L-DOPA+DMPO (in PBS, after 12 h); (C) LDOPA+DMPO (in PBS, after 24h); (D); L-DOPA+DMPO (in MiliQ water, after $24 \mathrm{~h}$ ); (E) L-DOPA + DMPO + deferoxamin ( $2 \mathrm{~mm})$; (F) L-DOPA + DMPO + $\%$ ethanol; (G) L-DOPA+DMPO $+20 \%$ ethanol. In all experiments; $200 \mu \mathrm{M}$ L-DOPA and $2.5 \%$ DMPO were used.

DMPO and hydroxyl radical, we studied the generation mechanism of hydroxyl radical using several conditions. As shown in Fig. 4, with the addition of hydrogen peroxide (Fig. 4B), the signal increased in a dose-dependent manner, while it was decreased under argon air (Fig. 4C); the addition of catalase inhibited the increment of ESR peaks of DMPO-OH (Fig. 4E), however, and superoxide dismutase had no appreciable effect (Fig. 4D). From these results, hydrogen peroxide and oxygen were thought to be involved in the generation mechanism of hydroxyl radical, and this generating system did not concerned superoxide anion. ${ }^{25)}$

Relative signal intensities of the spin adduct DMPO-OH, produced by hydroxyl radicals through autoxidation of LDOPA, decreased upon the addition of eugenol $\left(k_{\text {eugenol }+\cdot \mathrm{OH}}=\right.$ $8.3 \times 10^{9} \mathrm{M}^{-1} \mathrm{~s}^{-1}$ ) in a dose-dependent manner (Fig. 5). However, there remains some possibility of either trapping of hy- 
(A)

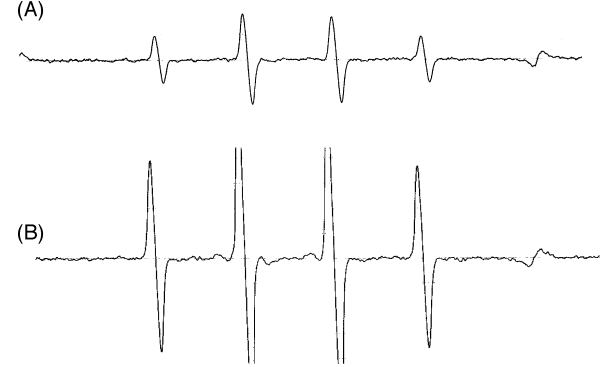

(C)
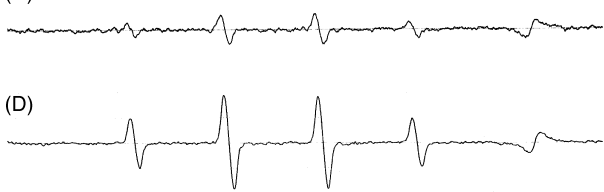

(E)

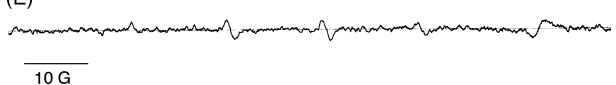

Fig. 4. ESR Spectra of Hydroxyl Radicals Produced by Incubation of LDOPA with DMPO

(A) L-DOPA + DMPO (control); (B) L-DOPA + DMPO+hydrogen peroxide $(0.1 \%)$ (C) L-DOPA+DMPO (under the argon air); (D); L-DOPA+DMPO+superoxide dismutase $(300 \mathrm{mU} / \mathrm{ml})$; (E) L-DOPA + DMPO + catalase $(100 \mathrm{mU} / \mathrm{ml})$. In all experiments; $200 \mu \mathrm{M} \mathrm{L}$-DOPA and 2.5\% DMPO were used. After adding L-DOPA, the ESR spectra were repetitively acquired for $24 \mathrm{~h}$. All compounds were dissolved in MiliQ water.

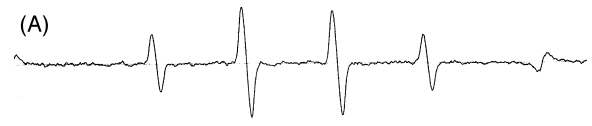

(B)

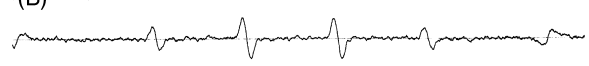

(C)

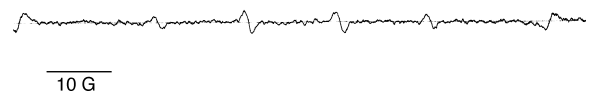

Fig. 5. Trapping Effect of Eugenol on Generating Hydroxyl Radical by LDOPA

(A) $\mathrm{L}-\mathrm{DOPA}+\mathrm{DMPO}$; (B) $\mathrm{L}-\mathrm{DOPA}+\mathrm{DMPO}+500 \mu \mathrm{M}$ eugenol; (C) $\mathrm{L}-\mathrm{DOPA}+$ $\mathrm{DMPO}+1 \mathrm{~mm}$ eugenol. All experiments: $200 \mu \mathrm{M}$ L-DOPA and $2.5 \%$ DMPO used. After adding L-DOPA, the ESR spectra were repetitively acquired for $24 \mathrm{~h}$.

droxyl radicals or of DMPO-OH itself. The reaction mixture contained L-DOPA and DMPO in MiliQ water and was incubated for $20 \mathrm{~h}$. After incubation of the reaction mixture eugenol was added and ESR spectra were recorded. Eugenol did not decrease the signal of DMPO-OH (data not shown).

L-DOPA has been thought to possibly produce semiquinone radical, and it may damage the cellular integrity causing cell death. ${ }^{26)}$ Therefore, we studied generation of the semiquinone radical under acidic, neutral and basic $\mathrm{pH}$ conditions. The semiquinone radical was stable under the basic condition (Fig. 6C) and was observable on ESR spectra, but not in an acidic or neutral condition (Figs. 6A, B). Haque et al. studied the semiquinone radical generation by ESR after 1 min incubation. ${ }^{27}$ In the present study, no generation of semiquinone radicals was observed in the experiments under a neutral condition after $24 \mathrm{~h}$. We examined whether eugenol also trapped the semiquinone radical, and found that at $1 \mathrm{~mm}$ it did not trap this radical under the basic $\mathrm{pH}$ condition used
(A)

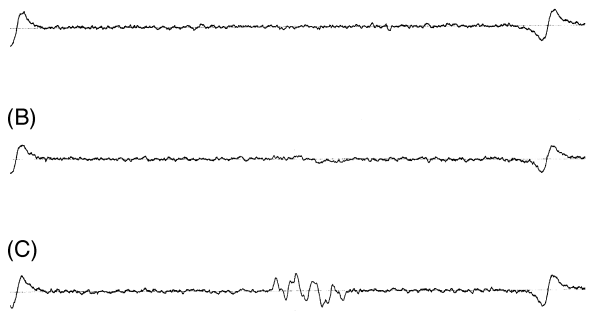

(D)

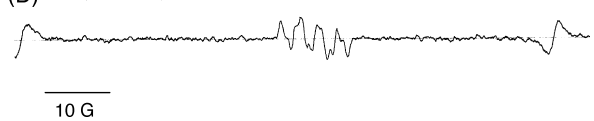

Fig. 6. ESR Spectra of L-DOPA Semiquinone Radicals

(A) $\mathrm{pH} 3$; (B) $\mathrm{pH} 7$; (C) $\mathrm{pH} 12$; (D) L-DOPA+1 mm eugenol (pH 12). All experiments used $200 \mu \mathrm{M}$ L-DOPA. After adding L-DOPA, the ESR spectra were repetitively acquired for $24 \mathrm{~h}$.

(Fig. 6D). This indicated that eugenol had a direct scavenging effect on the hydroxyl radicals, because it had no iron chelating action nor trapped L-DOPA semiquinone radical and inhibited hydroxyl radicals with or without iron ion.

In summary, we developed a generation system of the hydroxyl radical without using any metals by adding L-DOPA and DMPO to PBS or MiliQ water in vitro. We found that eugenol trapped hydroxyl radicals directly and is metabolized to dimer in vitro. ${ }^{20)}$ Detailed studies on biological activities of eugenol and its dimer are now under way.

Acknowledgments This work was supported in part by a research grant from KANPO Science Foundation, Tokyo Japan, Ministry of Education, Culture, Sports, Science and Technology, Japan and NEDO (New Energy and Industrial Technology Development Organization).

\section{References}

1) Reiss U., Gershon D., Biochem. Biophys. Res. Commum., 73, 255262 (1967).

2) Urano S., Hoshi-Hashizume M., Tochigi., Matsui M., Shiraki M., Ito H., Lipids, 26, 58-61 (1976).

3) Sun Y., Free Radic. Biol. Med., 8, 583-599 (1990).

4) Root R. K., Cohen M. S., Rev. Infect. Dis., 3, 565-598 (1981).

5) Henning B., Chow C. K., Free Radic. Biol. Med., 4, 99-106 (1988).

6) Halliwell B., Gutteridge J. M. C., Arch. Biochem. Biophys., 246, 501514 (1986).

7) Gutteridge J. M. C., Biochim. Biophys. Acta, 917, 219-223 (1987).

8) Halliwell B., FEBS Lett., 92, 321-328 (1978).

9) Gutteridge J. M. C., FEBS Lett., 201, 291-295 (1986).

10) Baehner R. L., Boxer L. A., Ingraham, L. M., "Free Radicals in Biology," Vol. 5, ed. by Pryor W. A., Academic Press, New York, 1982, pp. $91-127$

11) Torrielli M. V., Dianzai M. U., "Free Radicals in Molecular Biology, Aging, and Disease," Vol. 27, ed. by Armstrong D., Sohal R. S., Cutler R. G., Slater T. F., Raven Press, New York, 1984, pp. 355-379.

12) Lown J. W., Joshua A. V., "Free Radicals, Lipid Peroxidation, and Cancer," ed. by McBrien D. C. H., Slater T. F., Academic Press, New York, 1982, pp. 53-73.

13) Youngman R. J., Gotz F., Elstner E. F., "Oxygen Radicals in Chemistry and Biology," ed. by Bors W., Saran M., Tait D., Walter de Gruyter, Berlin, 1984, pp. 131-135.

14) Youngman R. J., Gotz F., Elstner E. F., "Oxygen Radicals in Chemistry and Biology," ed. by Bors W., Saran M., Tait D., Walter de Gruyter, Berlin, 1984, pp. 501-506.

15) Hutchinson F., Prog. Nucleic Acid Res. Mol. Biol., 32, 115-154 (1985).

16) Finkelstein E., Rosen G. M., Rauckman E. J., Mol. Pharmacol., 21, $262-265$ (1982). 
17) Walling C., Acc. Chem. Res., 8, 125-131 (1971).

18) Nagababu E., Lakshmaia N., Biochem. Pharmacol., 43, 2393-2400 (1992).

19) Fujisawa S., Atsumi T., Kadoma Y., Sakagami H., Toxicology, 177, $39-54$ (2002).

20) Ogata M., Hoshi M., Urano S., Endo T., Chem. Pharm. Bull., 48, 1467-1469 (2000).

21) Ko F. N., Liao C. H., Kuo Y. H., Lin Y. L., Biochim. Biophys. Acta, 1258, 145-152 (1995).

22) Basma A., Morris E. J., Nicklas J. W., Geller H. H., J. Neurochem., 64,
$825-832$ (1995).

23) Floyd A. R., Wiseman B. B., Biochim. Biophys. Acta, 586, 196-207 (1979).

24) Hoebeke M., Schuitmarker H. J., Dubbelman T. M., Jakobs A., Vandevorst A., Photochem. Photobiol., 66, 502-508 (1997).

25) Finkelstein E., Rosen G. M., Rauckman E. J., Arch. Biochem. Biophys., 200, 1-16 (1980).

26) LaVoie L. M., Hastings G. T., J. Nurosci., 19, 1484-1491 (1999).

27) Haque E. M., Asanuma M., Higashi Y., Miyazaki I., Tanaka K., Ogawa N., Biochim. Biophys. Acta, 1619, 39-52 (2003). 